\title{
Audiological findings in a group of neurologically compromised children: A retrospective study
}

\author{
K Baillieu, ${ }^{1}$ MScMed (Child Health Neurodevelopment); K Khoza-Shangase, ${ }^{1} \mathrm{PhD}$ (Audiology); \\ L Jacklin, ${ }^{2}$ FCP (Paed) (SA), MMed (Paed) \\ Department of Speech Pathology and Audiology, Faculty of Humanities, School of Human and Community Development, \\ University of the Witwatersrand, Johannesburg, South Africa \\ ${ }^{2}$ Department of Paediatrics, Faculty of Health Sciences, University of the Witwatersrand, Johannesburg, South Africa
}

Corresponding author: K Baillieu (kbaillieu@gmail.com)

\begin{abstract}
Background. Hearing loss is more prevalent in developing countries. Later diagnosis of hearing loss will result in delayed access to rehabilitation. It is typically more difficult to obtain subjective information required in a hearing test from neurocompromised children, causing audiologists to frequently turn to objective measures such as the auditory brainstem response (ABR) measure to obtain this information.

Objective. To describe the ABR results in a group of neurologically compromised children and to establish a relationship between ABR findings and behavioural audiometry results, where these existed.

Methods. A retrospective review was conducted on $40 \mathrm{ABR}$ patient records of neurologically compromised participants aged 5 months to 10 years. Behavioural audiometry results were sought where these existed. Hearing status was described per ear for objective and behavioural results, and descriptive statistics were conducted.

Results. Behavioural audiometry results were obtained in $72.5 \%$ of ears. Results correlated between ABR and behavioural audiometry for only $7.5 \%$ of ears, which were all diagnosed with normal hearing. About $12.5 \%$ of ears were misdiagnosed with behavioural audiometry. Premature infants were most likely to cope with behavioural audiometry. Hearing loss was highest in participants with cerebral palsy, Down syndrome, prematurity and retroviral disease.

Conclusions. Behavioural audiometry appears to be a largely unreliable method of testing the hearing of children diagnosed with neurological disorders, as results were obtained in only $27.5 \%$ of the study sample; however, it remains the gold standard in paediatric hearing testing to evaluate the entire auditory system and provides information on how a child processes sound. Hearing thresholds should be established via objective testing. Conditioning should continue for a behavioural audiological test battery, with adaptations for the child's developmental ability.
\end{abstract}

S Afr J Child Health 2016;10(1):20-24. DOI:10.7196/SAJCH.2016.v10i1.936

\section{Background}

\section{Early detection of hearing loss}

Hearing loss is a common sensory disability, with 800000 babies born with or acquiring a hearing loss worldwide each year. ${ }^{[1]}$ Ninety percent of children diagnosed each year with a permanent bilateral hearing loss of $\geq 40 \mathrm{~dB}$ reside in developing countries such as South Africa (SA) ${ }^{[1]}$ In SA, the prevalence rate of hearing loss is estimated at 3/1 000 in the private healthcare setting and 6/1000 in public healthcare. ${ }^{[2]}$ These high numbers highlight the importance of early hearing detection and intervention (EHDI) in developing countries. Documented evidence of EHDI benefits includes better expressive speech and language outcomes, ${ }^{[1]}$ thus decreasing the burden of permanent hearing loss and the limitation of educational opportunities, and ultimately improving long-term outcomes. ${ }^{[2]}$

The Health Professions Council of South Africa (HPCSA) identified populations at risk of developing hearing loss, who should be screened at birth if they present with recognised conditions. ${ }^{[3]}$ Acquired, late-onset and progressive hearing losses are not identified at birth; therefore, the HPCSA recommends that children with certain conditions associated with hearing loss receive hearing screening between the ages of 29 days and 2 years. ${ }^{[3]}$

The implementation of the HPCSA's position statement on EHDI has not been successful for a number of reasons. In SA, $85 \%$ of the population access public healthcare. ${ }^{[2]}$ Only $7.5 \%$ of public hospitals offer infant hearing screening, and only one hospital has been reported to offer universal hearing screening, i.e. $<10 \%$ of babies born in SA are afforded the opportunity of newborn hearing screening. ${ }^{[1]}$ Certain factors, such as equipment, personnel constraints and burden of disease, which prioritise life-threatening conditions such as HIV/AIDS, are barriers to EHDI.

\section{Early detection of hearing loss and intervention}

Many medical conditions in children fall in the category 'neurodisability' - some conditions place affected individuals at higher risk for hearing impairment than the general population, e.g. Down syndrome (DS). ${ }^{[4]}$ Other neurological disorders may result in a speech and language delay. It is therefore vital that the hearing status of these children be established early so that the benefits of EHDI can be realised.

\section{Hearing function and neurological disorders}

Hearing loss is higher in the HIV-infected paediatric population than in the general paediatric population. ${ }^{[5]}$ Hearing loss has been found to be largely conductive as a result of chronic suppurative otitis media, ${ }^{[6]}$ although sensorineural hearing loss (SNHL) has also been documented in higher-resourced areas. ${ }^{[5]}$

Hearing loss is more common in children with DS than in those with other developmental disabilities and healthy children ${ }^{[4]}$ because the former have narrow ear canals ${ }^{[7]}$ and eustachian tube dysfunction. ${ }^{[7]}$

All children with autistic spectrum disorder (ASD) will manifest auditory-related dysfunction of some level. ${ }^{[8]}$ These dysfunctions can include hyperacusis (increased sensitivity to sound), delayed cortical responses to low-frequency sounds, disrupted encoding of simple sounds, difficulty listening in noisy environments, and deafness. ${ }^{[8]}$

Children with cerebral palsy (CP) can have any amount of accompanying hearing loss. ${ }^{[9]}$ Of the infants with birth asphyxia worldwide, approximately $3 \%$ have mild hearing loss, and a further 
$3 \%$ severe hearing loss. ${ }^{[10]}$ Similarly, 5 - 10\% of premature infants globally are diagnosed with some degree of hearing loss. ${ }^{[1]}$

With regard to children with hydrocephalus, $81.5 \%$ have some degree of hearing loss ${ }^{[12]}$ which can improve with surgery to decrease cranial pressure. ${ }^{[12]}$ Among a cohort of meningitic children in Kenya, 43.4\% presented with SNHL post meningitis, of whom $61 \%$ had mild to moderate hearing loss and $38.7 \%$ severe to profound loss. ${ }^{[13]}$

\section{Assessing hearing function in participants}

When testing children's hearing, it is preferable to use the cross-check principle, where a variety of tests are conducted and the results compared. ${ }^{[14]}$ Behavioural audiometry allows threshold estimation across the entire frequency range for both air and bone conduction, which is essential information for speech and language development. ${ }^{[15]}$ Behavioural audiometry also provides an estimate of the entire auditory system from the outer ear to the auditory cortex, thus providing information on cognitive development. ${ }^{[15]}$ Behavioural audiometry alone is not enough, as the results obtained may be misleading and result in misdiagnosis. ${ }^{[14]}$ A test battery approach is reportedly $20 \%$ better to diagnose hearing loss than a single test. ${ }^{[14]}$

\section{Assessing hearing in special needs populations}

Owing to the neurological challenges influencing the ability of children with neurological disorders to be tested behaviourally, objective testing such as the auditory brainstem response (ABR) measure is recommended. ${ }^{[15]}$

The developmental age of the child has an effect on the success of the behavioural audiometry attempt. ${ }^{[16]}$ For children with special needs, certain adaptations can be made to the test battery and tasks to accommodate their needs. ${ }^{[17]}$ Positioning, test stimuli and the required response can be altered, based on motor and play skills of the child. ${ }^{[17]}$

According to the American Speech and Hearing Association (ASHA), for children with developmental disabilities, the diagnosis of hearing loss should rely primarily on objective testing, such as with the ABR. ${ }^{[18]}$ However, immittance audiometry, otoacoustic emissions, a comprehensive case history, behavioural observation, and functional hearing measures should all be conducted or at least attempted to supplement the ABR results. ${ }^{[18]}$

The ABR has been shown to provide a reliable estimate of hearing thresholds for the paediatric population. ${ }^{[18]}$ To optimise the efficiency of the test, different testing protocols may be used so that maximum information regarding the child's hearing status is obtained in the shortest possible time. ${ }^{[19]}$
Studies have shown that children with neurological disorders have ABRs that differ from those of children with normal neurological function. ${ }^{[20]}$ Children with ASD generally present with prolonged latencies of wave $\mathrm{V}$ and interpeak latencies. ${ }^{[20]}$ The ABR of children with DS has been shown to present with shorter latencies and lower amplitudes. ${ }^{[21]}$ In severe perinatal asphyxia, amplitudes of all waves - particularly wave $\mathrm{V}$ - were reduced in the first month of life. ${ }^{[22]}$ In a study in children with hydrocephalus, $88 \%$ of the cohort displayed a wide range of abnormalities on ABR. ${ }^{[23]}$

Similarly, children with CP presented with prolonged latencies on ABR testing. ${ }^{[24]}$ Children with meningitis were found to have increased interpeak latencies for waves I - V and I - III, and $14.8 \%$ had no visible waveforms at the maximum output of the equipment. ${ }^{[25]}$

These differences should therefore be taken into account when analysing the results in neurologically compromised children; hence the current retrospective review of audiological findings in a group of neurologically compromised children.

\section{Objectives}

The primary objective of this study was to describe the hearing test results in a group of neurologically compromised participants. The objectives were to:

- describe the audiological ABR findings to determine hearing function in this group

- compare audiological ABR and behavioural audiometry findings where these existed

- describe hearing results per pathological condition.

\section{Methods}

After obtaining the relevant permission, a descriptive retrospective review of patient records was conducted. Permission to review records was obtained from the relevant authorities, and data collection began only after permission from the pertinent review and ethics committees (protocol No. M120217). The first 40 audiological records of participants between the ages of 5 months and 10 years at a tertiary hospital in Johannesburg, SA were selected. All testing conducted for patient records was performed by qualified audiologists registered with the HPCSA.

Participants were tested during natural sleep, i.e. without sedation, at the tertiary hospital, with one or more of the following disorders:

- $\mathrm{CP}$

- developmental delay

- HIV/AIDS

- hydrocephalus

- DS

- birth asphyxia

- ASD

- meningitis.

The hearing testing protocol for paediatric patients is depicted in Fig. 1. Participants in the study had their hearing tested according to this protocol.

All hearing results for each participant were documented, including ABR and behavioural audiological testing if available. In cases of multiple attempts at behavioural audiometry, the most recent results were used. Each participant's results were described per ear, based on the click and tone-burst ABR thresholds documented on the records. The classification by Gelfand ${ }^{[26]}$ (Table 1) was used to describe the degree of hearing loss, based on the ABR threshold and behavioural results, using headphones, where available. Correction factors used in ABR testing are depicted in Table 2. Normal hearing in at least one cochlea was

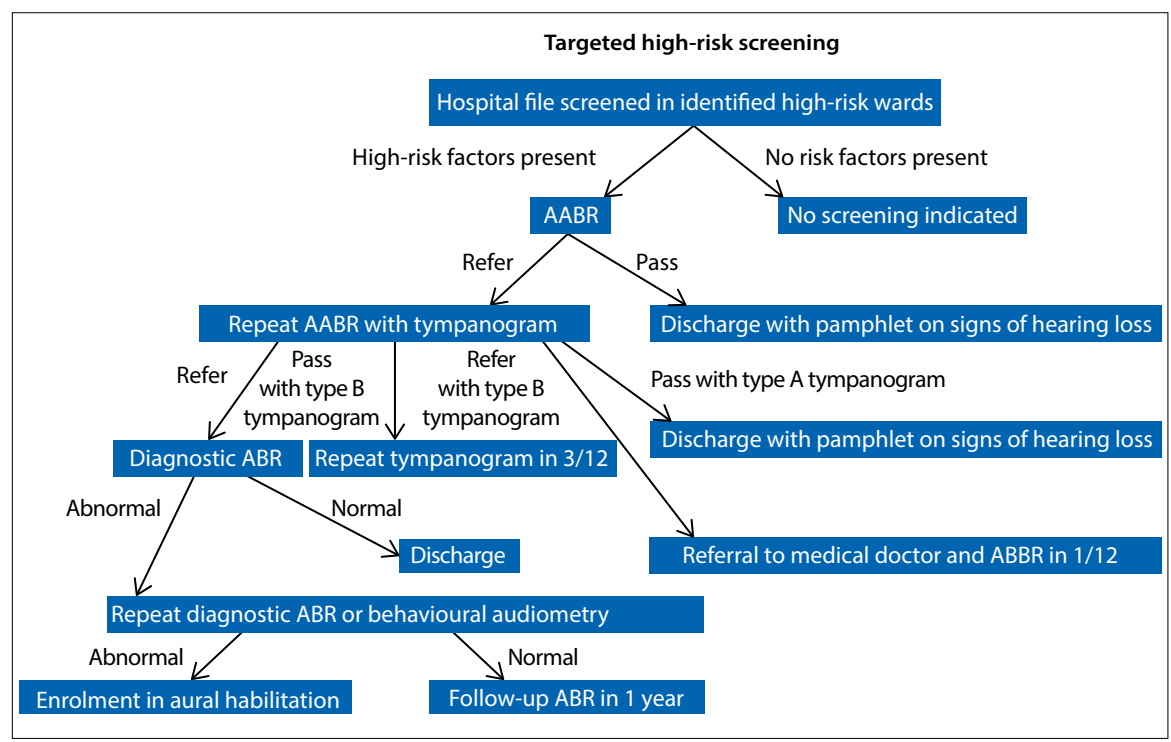

Fig. 1. Protocol for the paediatric hearing test $(A A B R=$ automated auditory brainstem response $)$. 
classified as $\leq 25 \mathrm{~dB}$ hearing loss on freefield testing. For participants tested in free field, overall hearing status was described and one ear was marked 'no results'.

Descriptive statistics were conducted per ear for all 40 participants in terms of the incidence and degree of hearing loss, which were compared with the ABR and behavioural results, where available. The frequency of the different neurological diagnoses was established and the hearing results of the six most frequent conditions were analysed according to the degree of hearing loss.

\section{Results}

The records of 21 males and 19 females were drawn, the average age being 28 months (standard deviation (SD) 24 (5 - 115) months).

As depicted in Table 3, of the records drawn for 40 participants, $62.5 \%$ presented with bilateral normal hearing, 30\% with bilateral symmetrical hearing loss, $20 \%$ with an asymmetrical hearing loss, and one participant was diagnosed with a unilateral hearing loss. In all participants hearing loss was diagnosed with $A B R$ testing, and some results of patients with normal hearing were corroborated on behavioural testing. One participant presented with a bilateral profound hearing loss when tested behaviourally; however, ABR testing indicated a lesserdegree bilateral asymmetrical hearing loss.

Analysis per ear indicated that for 58 of 80 ears $(72.5 \%)$, no behavioural audiological results had been obtained. Therefore, for $60 \%$ of the participants in this study, no behavioural results were obtained in either ear, and in $25 \%$ unilaterally. Half of the 16 participants for whom behavioural results were available

\begin{tabular}{ll}
$\begin{array}{l}\text { Table 1. Classification of degree of } \\
\text { hearing loss }\end{array}$ \\
\hline $\begin{array}{ll}\text { Pure-tone } \\
\text { average }(\mathrm{dB})\end{array}$ & Degree of hearing loss \\
\hline$<15$ & Normal hearing \\
$16-25$ & Slight hearing loss \\
$26-40$ & Mild hearing loss \\
$41-55$ & Moderate hearing loss \\
$56-70$ & $\begin{array}{l}\text { Moderately severe hearing } \\
\text { loss }\end{array}$ \\
$71-90$ & Severe hearing loss \\
$\geq 90$ & Profound hearing loss
\end{tabular}

Table 2. Correction values for ABR using earphones ${ }^{[27]}$

\begin{tabular}{lllll}
\hline Click & $500 \mathrm{~Hz}$ & $1 \mathrm{kHz}$ & $2 \mathrm{kHz}$ & $4 \mathrm{kHz}$ \\
\hline-5 & 15 & 10 & 5 & 0
\end{tabular}

presented with normal hearing. Three of these presented with normal hearing bilaterally using headphones; five were tested in free field and therefore had normal hearing in at least one cochlea.

\section{ABR compared with behavioural results}

Behavioural results were obtained in only 22 of the 80 ears tested. As four of the participants were aged between 5 and 6 months, behavioural audiometry was not attempted owing to their developmental capabilities. For six participants behavioural audiometry was aborted, and for a further six testing occurred in free field - therefore no earspecific results could be obtained.

Half of the ears for which there were behavioural results, were diagnosed with a hearing loss v. only $44 \%$ on ABR testing. In the sample of 80 ears tested, only $7.5 \%$ were diagnosed with normal hearing on both behavioural and objective testing. For 58 ears, no behavioural results were obtained, and for the remaining 16 ears there was a discrepancy between behavioural and $A B R$ results. Of these, $68.8 \%$ presented with worse hearing behaviourally than on objective testing. Therefore, $92.5 \%$ of the results differed according to behavioural and objective testing, and those that did correlate were in normal hearing ears only.

\section{Results per neurological condition}

The two most common diagnoses were prematurity (27.5\%) and CP (25\%). Hydrocephalus (17.5\%), DS (12.5\%), meningitis (12.5\%) and birth asphyxia (10\%) were also common, yielding a combined $52.5 \%$.

More hearing losses were diagnosed on ABR testing, with a total of 22 unilateral or bilateral hearing losses being diagnosed, as depicted in Fig. 2. Only six losses were diagnosed on the same sample with behavioural audiometry. A majority of participants with CP, DS, prematurity and retroviral disease were diagnosed with a hearing loss, as 19 of a combined total of 28 with these conditions presented with a hearing loss objectively. Additionally, 18 participants were diagnosed with normal hearing on ABR testing v. 7 on behavioural audiometry.

The majority of participants with hydrocephalus (6 of 7) and meningitis (3 of 5) were diagnosed with normal hearing on ABR testing. For the six main conditions, the participants with DS and HIV could not be tested behaviourally. Seven of 10 participants with CP, 3 of 5 with meningitis, 4 of 7 with hydrocephalus and 6 of 11 with prematurity could not undergo behavioural audiometry reliably.

\section{Discussion \\ Diagnosis of hearing status}

The responsiveness of a developmentally delayed participant is associated with their

Table 3. Hearing status in the sample $(N=40)$

\begin{tabular}{lll}
\hline Hearing status & Occurrence, $\boldsymbol{n}$ (\%) & Test method \\
\hline Normal hearing & $25(62.5)$ & $22 \mathrm{ABR}$ only, 5 both, 3 behavioural only \\
Bilateral symmetrical hearing loss & $12(30)$ & $10 \mathrm{ABR}$ only, 2 behavioural only \\
Bilateral asymmetrical hearing loss & $8(20)$ & $7 \mathrm{ABR}$ only, 1 behavioural only \\
Unilateral hearing loss & $1(2.5)$ & $1 \mathrm{ABR}$ only, 0 behavioural only
\end{tabular}

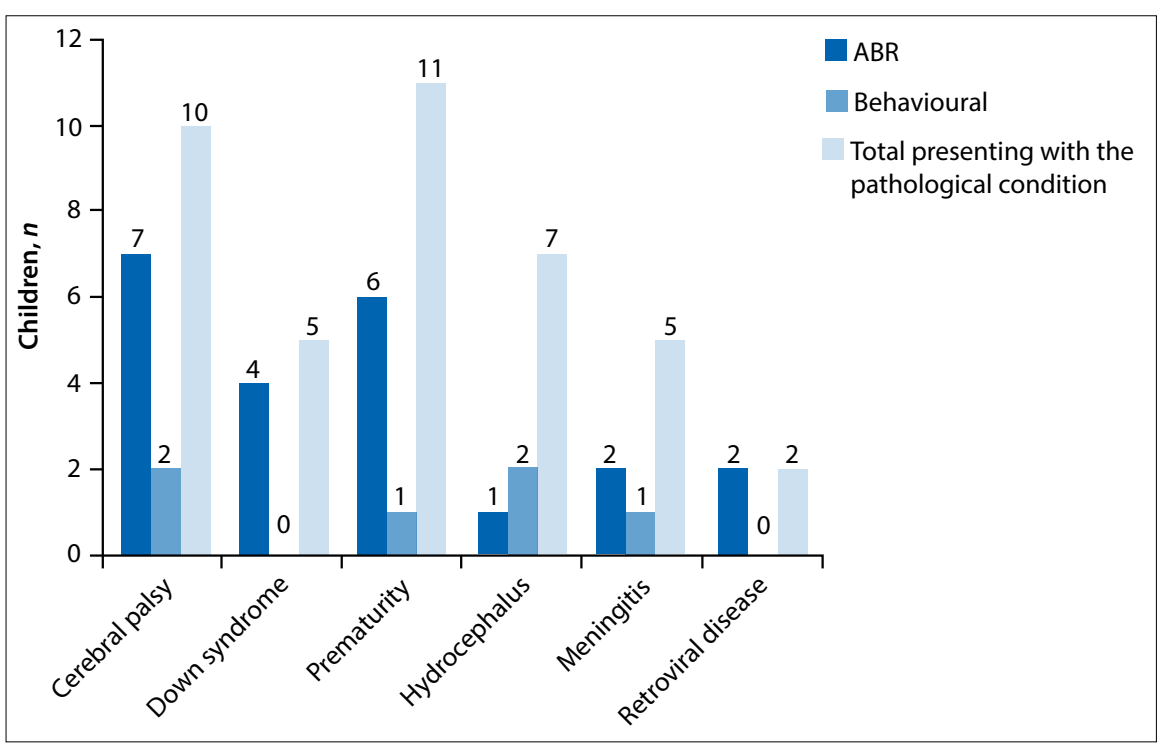

Fig. 2. Number of hearing losses diagnosed on ABR and behavioural audiometry for the six main conditions. 
developmental level, leading to both high false-positive and high false-negative results in behavioural audiometry. ${ }^{[17]}$ In our study, more hearing losses were diagnosed on ABR testing than with behavioural audiometry, indicating a difference in results of the two methods. One participant presented with a profound hearing loss bilaterally on behavioural audiometry and to a lesser degree on ABR testing. If the diagnosis of profound hearing loss was accepted, the participant's hearing would have been inappropriately amplified with hearing aids, risking further damage to the auditory system. This discrepancy between behavioural and objective findings reiterates that objective testing is particularly important in diagnosing hearing loss in a population with neurological disorders.

Likewise, in only 6 of the 80 ears tested was there a correlation between behavioural and ABR results and all of these ears were of normal hearing. These results suggest that for this study population behavioural audiometry was less sensitive at diagnosing both hearing loss and normal hearing than ABR testing.

\section{Behavioural audiometry}

By estimating the participant's developmental age, behavioural audiometry could be conducted in the same manner as for normaldeveloping participants of the same developmental age. ${ }^{[16]}$ Current findings indicate that no behavioural results could be obtained for a majority in this sample; hence the importance of planning testing batteries based on developmental rather than chronological ages.

It has been well established that behavioural audiometry remains the gold standard in hearing testing, as it provides an estimate of the entire auditory system from the outer ear to the auditory cortex. ${ }^{[15]}$ In half of this study population behavioural audiometry was not attempted.

For a child with a neurological disorder to have the best chance of completing the task required for behavioural audiometry, adaptations to the test battery would enhance acquisition of more reliable results. ${ }^{[16]}$ Interpretation of the results should be holistic, ensuring that these are cross-checked.

\section{Difficulty in obtaining behavioural responses}

The results of this study correspond to the finding that a child's ability to attend to a stimulus and nature of the response are dependent on the level of neuromaturation. ${ }^{[15]}$ The child's difficulty in responding and the tester not recognising the response would lead to hearing thresholds appearing worse than the child's actual hearing abilities.

A greater number of ears with a profound hearing loss were diagnosed on ABR testing than on behavioural audiometry. None of these participants objectively diagnosed with a profound hearing loss had any behavioural results. This could be the result of incorrect conditioning below the ear threshold; therefore, the child will not learn the conditioned response. ${ }^{[16]}$ If a child does not appear to condition under headphones at loud intensities, vibrotactile conditioning with a bone oscillator should be attempted to ascertain whether this lack of response is a potential hearing loss or a result of developmental delay. ${ }^{[16]}$ No participant in this study objectively diagnosed with a profound hearing loss could be tested behaviourally, which emphasises the importance of correct conditioning. ${ }^{[16]}$

Audiology departments at state hospitals service large populations. Time pressure may therefore lead to audiologists aborting the test and sending the child for an ABR. The audiologist may not include the adaptations and correct conditioning techniques that allow a neurologically disordered child the best opportunity to respond in a behavioural hearing test.

\section{Hearing loss with regard to diagnosis}

It is important to know with which condition the child has been diagnosed, as this can assist the audiologist with regard to whether a hearing loss should be expected and what type of hearing loss to anticipate, should it correspond to the diagnosis. ${ }^{[16]}$ The audiologist will also be able to gauge what to expect with speech, language and auditory behaviours, and to estimate the expected cognitive and developmental age of the child. ${ }^{[17]}$ Objective results can also be analysed, taking these factors into account.

In this study sample almost half of the participants were diagnosed with a hearing loss on $A B R$ testing, which is higher than the incidence of hearing loss of $6 / 100$ live births in the general paediatric population.

In this study sample of 5 participants with DS, 4 presented with hearing loss, which is higher than the average of $46.1 \% \cdot{ }^{[9]}$ This could be a result of some participants being diagnosed with normal hearing on screening measures in a diagnostic hearing evaluation and therefore being discharged without the requirement for ABR testing. Participants obtaining 'refer' results from the hearing screening at the research site are booked for an ABR and will therefore have a higher chance of presenting with a hearing loss, as they have already been referred on hearing screening.

Of the 11 participants born prematurely, 6 were diagnosed with a hearing loss on ABR testing, which is higher than the global prevalence of $5-10 \%{ }^{[11]}$ The higher occurrence of hearing loss in this subgroup emphasises the need for newborn hearing screening. Universal newborn screening is the gold standard; however, a lack of resources has prevented the instigation of this screening in SA. ${ }^{[28]}$ High-risk or targeted screening should, based on this study, include all premature infants.

Prematurity yielded the smallest number of no responses in behavioural audiometry compared with the five other common conditions. This indicates that these participants are generally able to undergo behavioural audiometry, given extra time to allow for brain maturation. However, with the higher occurrence of hearing loss in this premature population than in the general paediatric population, hearing loss would then be diagnosed at a later stage if more time was allowed for maturation. Audiological habilitation for those infants later diagnosed with hearing loss would be less effective.

More than half of the study population with meningitis presented with hearing loss, which reiterates the need to test this population once a diagnosis of meningitis has been made.

The three conditions yielding the least behavioural results are those for which the highest percentage of hearing loss was diagnosed, i.e. CP, retroviral disease and DS. This therefore emphasises the importance of these participants having their hearing tested, and the use of objective testing in obtaining results.

\section{HPCSA risk factors for hearing loss}

The HPCSA have identified participants who are at risk of developing a hearing loss and therefore should receive a hearing screen. ${ }^{[2]}$ For this study sample, of the 40 participants selected, only 14 would have been at risk according to the HPCSA criteria. Of the 26 participants with conditions not recognised by the at-risk register, 9 were found to have a hearing loss. Therefore, $22.5 \%$ of participants with a hearing loss diagnosed in this study sample would not have received a hearing screen. These were participants with $\mathrm{CP}$, prematurity and birth asphyxia. This indicates the need to revise the criteria for targeted screening at the research site and the HPCSA position statement, so that it is in line with the Joint Committee on Infant Hearing guidelines, ${ }^{[29]}$ which include follow-up screening for all children admitted to the neonatal intensive care unit for $>5$ days.

\section{Early intervention and neurological disorder}

Current findings have shown that hearing loss is more prevalent in populations with neurological disorders than in the general paediatric population. The findings also prove that most of these participants are diagnosed from objective measures, as behavioural audiometry is unreliable in such participants. 
According to the HPCSA's position statement, ${ }^{[3]}$ the guidelines for early intervention of hearing loss services in SA for hospital-based settings recommend confirmation diagnostic testing by 3 months of age before enrolment in an early intervention programme before 6 months of age. In this study, not one participant met these criteria. For many of the participants behavioural audiometry was attempted two or three times before a referral to the ABR clinic. This would delay the confirmation of hearing loss by a number of months.

\section{Conclusion}

It is suggested that all patients with neurological disorders receive a hearing screen as soon as a diagnosis of the neurological disorder is made. Those who do not pass hearing screening should be referred directly for objective testing in order to start habilitation. Behavioural audiometry should then be attempted, as this is the only measure that gives an indication of the functioning of the entire auditory system up to the auditory cortex. ${ }^{[15]}$ The child should be conditioned during a number of appointments with the abovementioned adaptations to the conventional behavioural audiological test to meet their individual developmental needs. However, this should be supplementary to enrolment in an early intervention programme to afford the child the best possible language and communication development. Once behavioural results are obtained, amplification devices should be reprogrammed based on the results.

\section{References}

1. Friderichs N, Swanepoel D, Hall JW. Efficacy of a community-based infant hearing screening programme utilizing existing clinic personnel in Western Cape, South Africa. Int J Pediatr Otorhinolaryngol 2012;76:552-559.

2. Meyer ME, Swanepoel D, le Roux T, van der Linde M. Early detection of infant hearing loss in the private health care sector of South Africa. Int J Pediatr Otorhinolaryngol 2012;76:698-703.

3. Swanepoel D. Health Professions Council of South Africa Professional Board for Speech, Language and Hearing Professions: Early Hearing Detection and Intervention Programmes in South Africa - Position Statement. Pretoria: Health Professions Council of South Africa, 2007. http://www.hpcsa.co.za/ downloads/speech_education/early_hearing_detection_statement.pdf. (accessed 10 August 2013).

4. Abou-Elhamd KA, ElToukhy HM, Al-Wadaani FA. Syndromes of hearing loss associated with visual loss. Eur Arch Otorhinolaryngol 2014;271(4):635-636. [http://dx.doi.org/10.1007/s00405-013-2514-0]

5. Laughton B, Cornell M, Boivin M, van Rie A. Neurodevelopment in perinatally HIV-infected participants: A concern for adolescence. J Int AIDS Soc 2013;16(18603):1-11. [http://dx.doi.org/10.7448/IAS.16.1.18603]

6. Malt EA, Dahl RC, Haugsand TM, et al. Health and disease in adults with Down syndrome. Tidsskr Nor Laegeforen 2013;133(3):290-294.

7. Austeng ME, Akre H, Øverland B, Abdelnoor M, Falkenberg E, Kværner KJ. Otitis media with effusion in participants with Down's syndrome. Int J Pediatr Otorhinolaryngol 2013;77:1329-1332. [http://dx.doi.org/10.1016/j. ijporl.2013.05.027]
8. Kulesza RJ, Lukose R, Stevens LV. Malformation of the human superior olive in autistic spectrum disorders. Brain Res 2010;1367:360-371. [http://dx.doi. org/10.1016/j.brainres.2010.10.015]

9. Rosenbaum P, Paneth N, Leviton A, Goldstein M, Bax M. A report: The definition and classification of cerebral palsy. Dev Med Child Neurol 2007;49:8-14.

10. Berke J. Birth asphyxia and hearing loss. 2011. http://deafness.about.com/ od/medicalcauses/a/Birth_Asphyxia_And_Hearing_Loss.htm (accessed 2 February 2016).

11. Nour NM. Premature delivery and the millennium development goal. Rev Obstet Gynecol 2012;5(2):100-105.

12. Dixon JF, Jones RO. Hydrocephalus-associated hearing loss and resolution after ventriculostomy. Otolaryngol Head Neck Surg 2012;146:1037-1039. [http:// dx.doi.org/10.1177/0194599811431234].

13. Karanja BW, Oburra HO, Masinde P, Wamalwa D. Risk factors for hearing loss in participants following bacterial meningitis in a tertiary referral hospital. Int J Otolaryngol 2013;2013:354725. [http://dx.doi.org/10.1155/2013/354725]

14. Diefendorf AO, Wynne MK. Paediatric audiology: A test battery approach. In: Kent RD, ed. The MIT Encyclopedia of Communication Disorders. Cambridge, MA: Massachusetts Institute of Technology, 2004:520-522.

15. Swanepoel D, Ebrahim S. Auditory steady-state response and auditory brainstem response thresholds in participants. Eur Arch Otorhinolaryngol 2009;266(2):213-219.

16. Northern JL, Downs MP. Hearing in Participants. 5th ed. Philadelphia, USA: Lippincott Williams and Wilkins, 2002.

17. Madell JR. Evaluation of hearing in participants with special needs. In: Madell JR, Flexer C, eds. Paediatric Audiology. Diagnosis, Technology and Management. New York: Thieme, 2008:82-89.

18. Purdy SC, Kelly AS. Auditory evoked response testing in infants and participants. In: Madell JR, Flexer C, eds. Paediatric Audiology: Diagnosis, Technology and Management. New York: Thieme, 2008:132-145.

19. Newman CW, Sandridge SA. Diagnostic audiology. In: Hughs GB, Pensak ML, eds. Clinical Otology. 3rd ed. New York: Thieme 2007:109-121.

20. Kwon S, Kim J, Choe B-H, Ko C, Park S. Electrophysiologic assessment of central auditory processing by auditory brainstem responses in participants with autism spectrum disorders. J Korean Med Sci 2007;22(4):656-659.

21. Krẹcicki T, Zalesska-Kręcicka M, Kubiak K, Gawron W. Brain auditory potentials in children with Down syndrome. Int J Paediatr Otorhinolaryngol 2005;69(5):615-620.

22. Jiang ZD, Brosi DM, Shao XM, Wilkinson AR. Sustained depression of brainstem auditory electrophysiology during the first months in term infants after perinatal asphyxia. Clin Neurophysiol 2008;119:1496-1505.

23. Kraus N, Özdamar Ö, Heydemann PT, Stein L, Reed NL. Auditory brain-stem responses in hydrocephalic patients. Electroen Clin Neuro 1984;59:310-317.

24. Zhu Q, Wang T, Liang J. The evaluation of auditory brainstem response and auditory steady-state response in children with cerebral palsy. J Clin Otorhinolaryngol 2006;20(22):1018-1019.

25. Topcu I, Cüreoqlu S, Yaramiş A, et al. Evaluation of brainstem auditory evoked response audiometry findings in children with tuberculous meningitis a admission. Auris Nasus Larynx 2002;29(1):11-14.

26. Gelfand SA. Pure tone audiometry. In: Gelfand SA, ed. Essentials of Audiology. 3rd ed. New York: Thieme, 2009:127-157.

27. Stevens J, ed. Guidelines for Early Audiological Assessment and Managemen of Babies Referred from the Newborn Hearing Screening Programme (version 2.5). London, UK: NHS Newborn Hearing Screening Programme, 2011.

28. Delaroche M, Gavilan-Cellie I, Maurice-Tison S, Kpozehouen A, Dauman R. Is behavioural audiometry achievable in infants younger than 6 months of age? Int J Pediatr Otorhinolaryngol 2011;75:1502-1509.

29. Joint Committee on Infant Hearing. Position statement: Principles and guidelines for early hearing detection and intervention programmes. Pediatrics 2007;120(4):898-921. 